\title{
Hopf bifurcation and synchronization of a five-dimensional self-exciting homopolar disc dynamo using a new fuzzy disturbance-observer-based terminal sliding mode control
}

\author{
Zhouchao Wei ${ }^{1,2 *}$, Amin Yousefpour ${ }^{3}$, Hadi Jahanshahi ${ }^{4}$, \\ Uğur Erkin Kocamaz ${ }^{5}$, Irene Moroz ${ }^{6}$ \\ 1. School of Mathematics and Physics, China University of Geosciences (Wuhan), Wuhan, 430074, China \\ 2. Zhejiang Institute, China University of Geosciences, Hangzhou, Zhejiang 311305, China \\ 3. School of Machanical Engineering, College of Engineering, University of Tehran, Tehran, 14399-57131, Iran \\ 4. Department of Mechanical Engineering, University of Manitoba, Winnipeg, R3T 5V6, Canada \\ 5. Department of Computer Technologies, Vocational School of Karacabey, \\ Bursa Uludag University, 16700 Karacabey, Bursa, Turkey \\ 6. Mathematical Institute, University of Oxford, Oxford OX2 6GG, England
}

\begin{abstract}
Having found hidden hyperchaos in a 5D self-exciting homopolar disc dynamo, we study the existence of a Hopf bifurcation, which leads to unstable limit cycles bifurcating from a stable equilibrium. Hidden chaos with only stable equilibria can be observed from the Hopf bifurcation: a typical way to enable hidden attractors to be located. We then provide a new fuzzy controller, and a fast fuzzy disturbance observer, based on terminal sliding mode control for synchronization of the hyperchaotic system. Fuzzy inference is considered to weaken the chattering phenomena. Using Lyapunov stability theory, the stability of the closed-loop system is proved. Finally, simulations of synchronization are illustrated to show the efficient performance of the designed control method via external disturbances and dynamic uncertainties.
\end{abstract}

Keywords: Hidden chaotic attractors; Hopf bifurcation; Terminal sliding mode control; Fuzzy disturbance-observer; External disturbances.

\section{Introduction}

The Lorenz system is a well-known touchstone for modern chaos theory. As the first mathematical model of chaos, this system led to further thoughts about complex phenomena in the real world. Many scholars have tried to find a unified theory of chaos, and the standard form of typical chaotic systems, in order to study chaos theoretically, and so reveal the nature of chaos $[33,50]$.

Nevertheless, the question remains: how to classify chaotic systems in autonomous systems in a reasonable way. There are still no systematic results, or much research on this matter. However, we can study the classification of chaotic systems from the analysis of the mechanism of chaos generation and from engineering applications. Some scholars have shown hidden attractors to be an important feature of multistability in some chaotic systems $[2,3,6,14-16,19,23,26,39,42,43,47]$. Some famous models of dynamo action have been widely investigated in the literature, as a way to understand the generation and reversal of magnetic and astrophysical fields. Moffatt incorporated azimuthal eddy currents to exclude the magnetic field and proposed a heuristic model of the disk dynamo [21], which generates hidden chaos as found by Wei et al [40]. Furthermore, Wei et al. pointed out the coexistence of point attractors, limit

\footnotetext{
${ }^{*}$ Corresponding author. E-mail addresses: weizhouchao@163.com
} 
cycles, chaotic spiral attractors and hidden hyperchaos in a 5D self-exciting homopolar disc dynamo when a broad range of parameters vary [41].

On the other hand, some chaotic models, found in theoretical research, can also be widely used in practical problems. To date, many control techniques, including backstepping method [Yassen, 2007], adaptive control [12,13,29,35], fuzzy control [10,20,27,28], optimal control [32], and sliding mode control (SMC) [9-11] have been proposed for nonlinear and chaotic system. SMC as a robust control technique has attracted remarkable attention in various practical applications [37]. Recently, various studies have applied SMC for the control and synchronization of classic and hidden chaos [30]. Mobayen adopted a robust adaptive sliding mode control (ASMC) to improve the performance of a chaotic flow [22]. Aghababa and Heydari developed an ASMC to obtain chaotic synchronization between different systems [1]. The existence of unknown disturbances is undeniable in real applications. Therefore, a disturbance observer could dramatically improve the performance of the controller [17]. To this end, numerous studies have been proposed for disturbance-observer-based control methods [7,38, 49].

One of the major drawbacks to SMC is the unknown convergence time. Moreover, SMC does not guarantee the convergence of a system to the desired output in a finite time. To address this issue, researchers have proposed either terminal sliding mode control (TSMC) or integral terminal sliding mode to guarantee finite-time convergence of the closed-loop system $[36,46]$. In this regard, a neural networkbased TSMC has been proposed by Yousefpour et al. [45] for a hyper-chaotic system with transient chaos. $\mathrm{Ni}$ et al. [25] have proposed a fast fixed-time TSMC, and used this technique to suppress chaos in power systems. Using adaptive laws and finite-time stability theory, Sun et al. [34] have proposed a non-singular TSMC approach for synchronization of chaotic systems.

In the current study, TSMC is combined with a fast fuzzy disturbance observer for synchronization of a five-dimensional self-exciting homopolar disc dynamo. The current study solves some problems and improves the methods that are proposed in the previous studies for control and synchronization of MIMO nonlinear systems. The proposed controller has some advantages in comparison with its stateof-the-art counterparts. For instance, Huang has proposed a fast TSMC with novel fuzzy disturbance observer [8], in which the disturbance observation error is asymptotic convergence, and it may take a long time to estimation error become zero. In 2017, Nguyen et al. [24] have proposed a new fuzzydisturbance observer-enhanced SMC for vibration control of a train-car suspension. However, based on their techniques, systems do not converge to the desired points in finite time. In 2018, a disturbance observer-based fuzzy TSMC has been proposed by Liu et al. [18] for a hypersonic vehicle. Although in their study, the finite time convergence of disturbance observer and the closed-loop system has been proven, the chattering phenomenon is completely neglected, which lower the controller's effective performance. In their technique, both controller and observer may cause chattering. Though they have implemented a fuzzy logic engine to adjust a parameter in the control signal, they did not use the fuzzy engine's potential for chattering elimination. On the other hand, the sliding surface we propose in the current study is entirely different from that, and our control scheme is chatter-free. More recently, in a study, Vahidi-Moghaddam et al. [36] have offered an observer-based fuzzy TSMC for MIMO systems. However, the significant drawback in their controller is convergence time. They have only proven the finite time convergence of disturbance observer, but the controller is exponentially stable, and the finite time convergence is not guaranteed. Hence, in some cases, convergence time will be very long. On the other hand, in our controller finite time convergence of disturbance observer and controller are proven simultaneously.

We return to the dynamical analysis of the 5D self-exciting homopolar disc dynamo proposed by Wei et.al [41], to investigate Hopf bifurcations, leading to unstable limit cycles from the asymptotically stable equilibrium point. This will be useful for clarifying the emergence of chaos. In addition, the combination of TSMC, a fast disturbance observer and a fuzzy controller are designed for synchronizing the 5D selfexciting homopolar disc dynamo in the presence of disturbances. To prevent chattering in the response of the system and control input, a fuzzy logic engine is used with TSMC. Also, due to the fact that the fuzzy system adaptively updated the gains of the disturbance observer, the proposed fuzzy interference will increase the convergence speed of the system in comparison with the traditional TSMC. As far as we know, little research has been done about proposing a chatter-free fuzzy disturbance observer-based TSMC with guaranteed finite-time convergence of both disturbance observer and closed-loop system for MIMO systems. The designed controller is applied to the 5D self-exciting homopolar disc dynamo, and its performance has been demonstrated through numerical simulations.

The paper is organized as follows. In Sec. 2, we present the homopolar disc dynamo, study Hopf 
bifurcations from this state, and show the bifurcation of an unstable limit cycle. In Sec. 3, we present the fuzzy disturbance-observer-based TSMC and confirm convergence of the system in finite time. Sec. 4 contains numerical simulations of the synchronization. Finally, in Sec. 5, we summarize the results.

\section{System Description and Hopf bifurcation}

In [41], Wei et al. introduced a 5D self-exciting homopolar disc dynamo and discussed the presence of hidden chaotic and hyperchaotic attractors, as well as multistability. The system is given by the following set of five coupled first order ordinary differential equations:

$$
\left\{\begin{array}{l}
\dot{x}=r(y-x)+w \\
\dot{y}=-(1+m) y+x z-v \\
\dot{z}=g\left[1+m x^{2}-(1+m) x y\right] \\
\dot{w}=2(1+m) w+x z-k_{1} x \\
\dot{v}=-m v+k_{2} y
\end{array}\right.
$$

where $r, m, g$ are positive parameters, and $k_{1}, k_{2}$ are control parameters. For the parameters choice of $r=8, m=0.04, g=140.6, k_{1}=34$ and $k_{2}=12$, system (2.1) exhibits hidden hyperchaotic behavior, as shown in Fig. 1 and Fig.2. Further details can be found in [41].

Now we recall the method for finding the first Lyapunov coefficient $l_{1}$ to give the stability of a Hopf bifurcation [39] for the general system:

$$
\dot{X}=f(X, \mu),
$$

where $X \in R^{5}$ and $\mu \in R^{1}$. We assume that $f$ is in a $C^{\infty}$ class in $R^{5} \times R^{1}$. Suppose that (2.2) has an equilibrium $X=X_{0}$ at $\mu=\mu_{0}$, which we write as $X=X_{0}$ at $\mu=\mu_{0}$, and denoting the variable $X-X_{0}$ also by $X$. Then

$$
\begin{gathered}
F(X)=f\left(X, \mu_{0}\right), \\
F(X)=A X+\frac{1}{2} B(X, X)+\frac{1}{6} C(X, X, X)+o\left(\|X\|^{4}\right),
\end{gathered}
$$

where $A=f_{x}\left(0, \mu_{0}\right)$ and

$$
B(X, Y)=\left.\sum_{j, k=1}^{5} \frac{\partial^{2} F_{i}(\xi)}{\partial \xi_{j} \partial \xi_{k}}\right|_{\xi=0} X_{j} Y_{k}, \quad C(X, Y, z)=\left.\sum_{j, k, l=1}^{5} \frac{\partial^{3} F_{i}(\xi)}{\partial \xi_{j} \partial \xi_{k} \partial \xi_{l}}\right|_{\xi=0} X_{j} Y_{k} Z_{l}
$$

We take $T^{c}$ to be the generalized eigenvalues of $A$, with only one pair of complex eigenvalues on the imaginary axis, with no other eigenvalues having $\operatorname{Re} \lambda=0$. Let $p, q \in R^{5}$ be vectors such that

$$
A q=\omega i q, \quad A^{T} p=-\omega i p, \quad<p, q>=\sum_{i=1}^{3} \bar{p}_{i} q_{i}=\bar{p}_{1} q_{1}+\bar{p}_{2} q_{2}+\bar{p}_{3} q_{3}=1
$$

where $A^{T}$ is the transpose of the matrix. Any vector $y \in T^{c}$ can be expressed as $y=\omega q+\bar{\omega} \bar{q}$, where $\omega=\langle p, y\rangle \in C$. The two-dimensional center manifold, associated with the eigenvalues $\lambda_{2,3}$ can be parameterized by $\omega$ and $\omega$, through an immersion of the form $X=H(\omega, \bar{\omega})$, where $H: C^{2} \rightarrow R^{2}$ has a Taylor expansion of the form

$$
H(\omega, \bar{\omega})=\omega q+\bar{\omega} \bar{q}+\sum_{2 \leq j+k \leq 3} \frac{1}{j ! k !} h_{j k} \omega^{j} \bar{\omega}^{k}+o\left(|\omega|^{4}\right),
$$

with $h_{j k} \in R^{5}$ and $h_{j k}=h_{k j}^{-}$. Substituting (2.7) into (2.3), we get the differential equation

$$
H_{\omega} \omega^{\prime}+H_{\omega} \bar{\omega}^{\prime}=F(H(\omega, \bar{\omega})),
$$

where $F$ is given by (2.3) and (2.4). In addition, (2.4) can be written on the chart $\omega$ for a central manifold as

$$
\dot{\omega}=i \omega_{0} \omega+\frac{1}{2} G_{21} \omega|\omega|^{2}+o\left(|\omega|^{4}\right)
$$


with $G_{21} \in C$. The first Lyapunov coefficient is then given by

$$
l_{1}=\frac{1}{2} \operatorname{Re} G_{21},
$$

with

$$
G_{21}=\left\langle p, C(q, q, \bar{q}), B\left(\bar{q}, h_{20}\right)+2 B\left(q, h_{11}\right)\right\rangle .
$$

Setting the rhs of equations (2.1) to zero, we obtain the equilibrium state

$$
x^{2}=\frac{k_{2}+m+m^{2}-2 m r-2 m^{2} r}{k_{1} m-k_{2} m-m^{2}+k_{1} m^{2}-m^{3}-2 m r-2 m^{2} r},
$$

together with

$$
y=\frac{m x \Delta_{1}}{\Delta_{2}}, z=\frac{\left(k_{2}+m+m^{2}\right) \Delta_{1}}{\Delta_{2}}, u=\frac{k_{2}+m\left(1-k_{1}+m\right)}{\Delta_{2}} r x, v=-\frac{k_{2} x \Delta_{1}}{\Delta_{2}},
$$

where $\Delta_{1}=k_{1}-2(1+m) r, \Delta_{2}=k_{2}-m(1+m)(2 r-1)$. Therefore, when $\left(k_{2}-m(1+m)(2 r-1)\right)\left(k_{2}-\right.$ $\left.k_{1}(1+m)+(1+m)(m)+2 r\right)<0$, system $(2.1)$ has two equilibrium states: $A_{1}=\left(x_{1}, y_{1}, z_{1}, w_{1}, v_{1}\right), A_{2}=$ $\left(x_{2}, y_{2}, z_{2}, w_{2}, v_{2}\right)$, where $x_{1}$ and $x_{2}$ are the positive and negative roots of equation (2.12), respectively. Here, we choose parameters $k_{1}=34, r=8, g=140.6, k_{2}=12$, and take $m$ to be the bifurcation parameter. The characteristic equation about the equilibrium $A_{1}$ is then:

$$
\lambda^{5}+\delta_{1} \lambda^{4}+\delta_{2} \lambda^{3}+\delta_{3} \lambda^{2}+\delta_{4} \lambda+\delta_{5}=0,
$$

where

$$
\begin{aligned}
& \delta_{1}=7 \\
& \delta_{2}=-\frac{-33744+65736 m+149175 m^{2}-98515 m^{3}-144500 m^{4}-61520 m^{5}-835 m^{6}+75 m^{7}}{5 m\left(6-17 m+m^{2}\right)\left(-4+5 m+5 m^{2}\right)}, \\
& \delta_{3}=-\frac{-202464+446088 m-450312 m^{2}+18613 m^{3}+651545 m^{4}+272715 m^{5}+42625 m^{6}-260 m^{7}+50 m^{8}}{5 m\left(-6-17 m+m^{2}\right)\left(-4+5 m+5 m^{2}\right)}, \\
& \delta_{4}=-\frac{4218\left(36-14 m-163 m^{2}-61 m^{3}+21 m^{4}+5 m^{5}\right)}{5 m\left(-6-17 m+m^{2}\right)}, \\
& \delta_{5}=-\frac{4218}{5}\left(-4+5 m+5 m^{2}\right) .
\end{aligned}
$$

It is therefore not difficult to show that when parameter $m=0.14662$, equation (2.14) has a pair of pure imaginary roots, and no other roots having positive real parts. All the eigenvalues are: $\lambda_{1}=-0.136286$, $\lambda_{2,3}=-3.43186 \pm 2.56525 i, \lambda_{4,5}= \pm 32.63850 i$. The transversality condition is also satisfied since $\left.\operatorname{Re}\left(\lambda^{\prime}\left(m_{0}\right)\right)\right|_{\lambda=32.63850 i} \approx 9.37479>0$, and we obtain the following Theorem.

Theorem 2.1. When $r=8, m=0.04, g=140.6, k_{1}=34$, and $k_{2}=12$, as $m$ passes through the critical value $m=0.14662$, system (2.1) undergoes a Hopf bifurcation at the equilibrium states $A_{1,2}=( \pm 2.76242, \pm 0.66895,20.09670, \pm 16.74780, \pm 54.74860)$.

Theorem 2.2. When $m=0.14662$, the first Lyapunov coefficient, related to the equilibrium point $A_{1,2}$ is $l_{1}=-5.98732<0$, and the Hopf bifurcation points at $A_{1,2}$ are both weakly stable foci. Moreover, for $m<m_{0}=0.14662$, but close to $m_{0}$, there are unstable limit cycles around the asymptotic stable equilibrium points $A_{1,2}$.

Proof. The two equilibrium points $A_{1}$ and $A_{2}$ have the same stabilities because of symmetry. Here, we focus on one equilibrium point: $A_{1}$ to compute the first Lyapunov coefficient $l_{1}$ and so obtain its stability. From equation (2.5), we have the following the multi-linear symmetric functions

$$
\begin{aligned}
& B(x, y)=\left(0, x_{1} y_{3}+x_{3} y_{1}, g\left(2 m x_{1} y_{1}-(1+m)\left(x_{1} y_{2}+x_{2} y_{1}\right)\right), x_{1} y_{3}+x_{3} y_{1}, 0\right), \\
& C(x, y, z)=(0,0,0) .
\end{aligned}
$$


Furthermore, we also obtain

$$
\begin{aligned}
& p=\left(0.04402-0.15793 i, 0.59427_{0} .02237 i,-0.33455+8.10054 i, 0.75247-0.00581 i, 0.00921-0.21845 i\right), \\
& q=(0.11148+0.50438 i, 0.85277,-0.00020+0.07085 i,-0.0156+0.00232 i,-0.00012-0.02613 i), \\
& h_{11}=(-0.9305,-0.04675,-0.02586,-1.17039,-3.82601), \\
& h_{20}=(0.01667-0.00267 i, 0.03334+0.11781 i,-2.85748+0.84832 i, 0.04107+0.12413 i, 0.02167-0.00608 i), \\
& G_{21}=-11.9746-326.4400 i,
\end{aligned}
$$

and

$$
l_{1}=-5.98732 .
$$

Theorem 2.2 is therefore proved.

When initial conditions $(2.8,1,20,16.7,55)$, if we choose parameters $k_{1}=34, r=8, g=140.6$, $k_{2}=12$ and $m=0.142<m_{0}=0.14662$, we obtain an unstable limit cycle around $A_{2}=(2.83319,0.66136$, 19.99330, 17.37460, 55.88950), as shown in Fig. 3 (eliminating initial transients). For the initial conditions $(1,0,0,0,4.9)$, we obtain the chaotic attractor shown in Fig. 4. We therefore have multistability with the co-existence of a hidden chaotic attractor with an unstable limit cycle. Hidden chaos with only stable equilibria can be found from Hopf bifurcations, a typical way to enable hidden attractors to be located.

\section{Controller design}

Due to inherent unpredictability, investigation of chaotic systems in terms of stabilization and synchronization is an important subject of study. If the governing equation for a multiple-input multiple-output system takes the form;

$$
\dot{x}(t)=f(x)+\Delta f(x)+(g(x)+\Delta g(x)) u_{c}+d_{0}(t),
$$

where $x=\left[x_{1}, x_{2}, \ldots, x_{n}\right]^{T}$ is the state vector, and $f(x)$ and $g(x)$ are nonlinear functions that represent system dynamics. The column vectors

$$
u_{c}=\left[u_{c 1}, u_{c 2}, \ldots, u_{c n}\right]^{T}, \quad d_{0}=\left[d_{0_{1}}, d_{0_{2}}, \ldots, d_{0_{n}}\right]^{T}
$$

represent the control input and external disturbance, respectively, and $\Delta f$ and $\Delta g$ give the nonlinear functions that indicate the modeling uncertainties of the system. Taking the uncertain disturbance terms as a compound disturbance that yields term $d(t)$, the system can be written as

$$
\begin{aligned}
& \dot{x}(t)=f(x)+g(x) u_{c}+d(t), \\
& d(t)=\Delta f(q)+\Delta g(q) u_{c}+d_{0}(t) .
\end{aligned}
$$

The following two lemmas prove the stability and finite time convergence of disturbance observer.

Lemma 3.1. Suppose positive definite function $V(t)$ which fulfills the following inequality [48]:

$$
\dot{v}(t)+\vartheta V(t)+\xi V \chi \leq 0, \forall t>t_{0},
$$

where $\vartheta>\xi>0$ and $0<\chi<1$. Then, the convergence time of $V(t)$ is given by $t_{s}$,

$$
t_{s} \leq t_{0}+\frac{1}{\vartheta(1-\chi)} \ln \frac{\vartheta V^{1-\chi}\left(t_{0}\right)+\xi}{\xi} .
$$

Lemma 3.2. Using the triangle inequality: if $a_{\Delta}>0, \Delta=1,2, \ldots, m$, and $0<n<1$, then

$$
\left(\sum_{\Delta=1}^{m} a_{\Delta}\right)^{n} \leq \sum_{\Delta=1}^{m} a_{\Delta}^{n} .
$$




\subsection{Fast disturbance observer}

Variables $s_{d}$ and $z$ are introduced to design the terminal disturbance observer [Chen et al., 2013]:

$$
s_{d}=z-x,
$$

where $z$ is

$$
\dot{z}=-k_{d} s_{d}-\beta \operatorname{sign}\left(s_{d}\right)-\varepsilon s_{d} p_{0} / q_{0}-|f(x)| \operatorname{sign}\left(s_{d}\right)+g(x) u_{c} .
$$

Here $\beta>d_{1}$ and parameters $k_{d}$ and $\varepsilon$ are positive. Moreover, $q_{0}$ and $p_{0}$ are two odd positive integers with $p_{0}<q_{0}$. The disturbance observer $\hat{d}$ is then given by

$$
\hat{d}=-k_{d} s_{d}-\beta \operatorname{sign}\left(s_{d}\right)-\varepsilon s_{d} p_{0} / q_{0}-|f(x)| \operatorname{sign}\left(s_{d}\right)-f(x) .
$$

Using Eqs. (3.20), (3.24), and (3.25) we obtain

$$
\dot{s_{d}}=\dot{z}-\dot{x}=-k_{d} s_{d}-\beta \operatorname{sign}\left(s_{d}\right)-\varepsilon s_{d}{ }^{p_{0} / q_{0}}-|f(x)| \operatorname{sign}\left(s_{d}\right)-f(x)-d .
$$

Then Eqs. (3.20), (3.26), and (3.27) give

$$
\begin{aligned}
\tilde{d} & =\hat{d}-d=-k_{d} s_{d}-\beta \operatorname{sign}\left(s_{d}\right)-\varepsilon s^{p_{0} / q_{0}}-|f(x)| \operatorname{sign}\left(s_{d}\right)-f(x)-d \\
& =-k s_{d}-\beta \operatorname{sign}\left(s_{d}\right)-\varepsilon s_{d} p_{0} / q_{0}-|f(x)| \operatorname{sign}\left(s_{d}\right)-f(x)-\dot{x}+f(x)+g(x) u_{c} \\
& =-k s_{d}-\beta \operatorname{sign}\left(s_{d}\right)-\varepsilon s_{d}{ }^{p_{0} / q_{0}}-|f(x)| \operatorname{sign}\left(s_{d}\right)+g(x) u_{c}-\dot{x} \\
& =\dot{z}-\dot{x} \\
& =\dot{s_{d}} .
\end{aligned}
$$

Theorem 3.1. For the nonlinear system (3.20), using disturbance observer (3.24)-(3.26), the error of disturbance estimation tends to zero in the finite time.

Proof. Let the Lyapunov function be

$$
V_{0}=\frac{1}{2} s_{d}^{T} s_{d}
$$

The time derivative of $V_{0}$ is

$$
\begin{aligned}
\dot{V}_{0} & =s_{d}^{T} s_{d} \\
& =s_{d}^{T}\left(-k_{d} s_{d}-\beta \operatorname{sign}\left(s_{d}\right)-\varepsilon s_{d}{ }^{p_{0} / q_{0}}-f(x){ }_{1} \operatorname{sign}\left(s_{d}\right)-f(x)-d\right) \\
& \leq-k_{d} s_{d}^{T} s_{d}-\beta s_{d}^{T} \operatorname{sign}\left(s_{d}\right)-\varepsilon s_{d}^{T} s_{d}^{p_{0} / q_{0}}-f(x){ }_{1} s_{d}^{T} \operatorname{sign}\left(s_{d}\right)-s_{d}^{T} f(x)-s_{d}^{T} d \\
& \leq-k_{d} s_{d}^{T} s_{d}-\beta s_{d}^{T} 1-\varepsilon s_{d}^{T} s_{d}^{p_{0} / q_{0}}-f(x){ }_{1} s_{d}^{T} 1-s_{d}^{T} f(x)+s_{d}^{T} 1 d_{1} \\
& \leq-k_{d} s_{d}^{T} s_{d}-\varepsilon s_{d}^{T} s_{d}^{p_{0} / q_{0}} \\
& \leq-2 k V_{0}-2^{\left(p_{0}+q_{0}\right) / 2 q_{0}} \varepsilon V_{0}^{\left(p_{0}+q_{0}\right) / 2 q_{0}} .
\end{aligned}
$$

Eqs. (31)-(33) show how the last line of Eq. (3.30) has been obtained.

$$
\begin{aligned}
& V_{0}=\frac{1}{2} s_{d}^{T} s_{d}=\frac{1}{2}\left(s_{d 1}^{2}+s_{d 2}^{2}+\ldots+s_{d n}^{2}\right), \\
& V_{0}^{\left(p_{0}+q_{0}\right) / 2 q_{0}}=\left(\frac{1}{2}\left(s_{d 1}^{2}+s_{d 2}^{2}+\ldots+s_{d n}^{2}\right)\right)^{\left(p_{0}+q_{0}\right) / 2 q_{0}} .
\end{aligned}
$$

Hence, the following inequality has been obtained

$$
2^{\left(p_{0}+q_{0}\right) / 2 q_{0}} V_{0}^{\left(p_{0}+q_{0}\right) / 2 q_{0}} \leq s_{d 1}\left(p_{0}+q_{0}\right) / q_{0}+s_{d 2}\left(p_{0}+q_{0}\right) / q_{0}+\ldots+s_{d n}{ }^{\left(p_{0}+q_{0}\right) / q_{0}} .
$$

Also, since $s_{d 1}\left(p_{0}+q_{0}\right) / q_{0}+s_{d 2}{ }^{\left(p_{0}+q_{0}\right) / q_{0}}+\ldots s_{d n}{ }^{\left(p_{0}+q_{0}\right) / q_{0}}=s_{d}{ }^{T} s_{d}{ }^{p_{0} / q_{0}}$, we have

$$
\begin{array}{r}
2^{\left(p_{0}+q_{0}\right) / 2 q_{0}} V_{0}^{\left(p_{0}+q_{0}\right) / 2 q_{0}} \leq s_{d}^{T} s_{d} p^{p_{0} / q_{0}} \\
\rightarrow-\varepsilon s_{d}{ }^{T} s_{d}{ }^{p_{0} / q_{0}} \leq-\varepsilon 2^{\left(p_{0}+q_{0}\right) / 2 q_{0}} V_{0}^{\left(p_{0}+q_{0}\right) / 2 q_{0}} .
\end{array}
$$


Based on Lemma 3.1, Lemma 3.2 and Eq. (3.30), the error of the disturbance observer $(\tilde{d})$ converges to zero in finite time, given by:

$$
t_{s \Delta}<t_{0 \Delta}+\frac{q_{0}}{k\left(q_{0}-p_{0}\right)} \ln \left(\frac{k s_{0 \Delta}^{\left(q_{0}-p_{0}\right) / 2 q_{0}} t_{0 \Delta}}{\varepsilon}+1\right), \Delta=1,2, \ldots, n
$$

where $t_{s \Delta}$ is the convergence time and $t_{0 \Delta}$ is the initial time.

\subsection{TSMC}

Using the disturbance observer developed in section 3.1, the TSMC is designed for system (3.20). The tracking error of the system is given by

$$
e(t)=\left[e_{1}(t), e_{2}(t), \ldots, e_{n}(t)\right]^{T}=x(t)-x_{d}(t),
$$

where $x_{d}(t)$ is the required value for the state $x(t)$. We define the terminal sliding surface as

$$
s_{t}(t)=e(t)+s_{d}(t)
$$

where $s_{d}(t)$ is the auxiliary variable, given in equation (3.24). Finally, the disturbance-observer-based TSMC for system (3.20) is taken to be

$$
u_{c}=-g^{-1}(x)\left(f(x)-\dot{x}_{d}+\delta s_{t}+\zeta s_{t}^{p / q}+\hat{d}\right),
$$

where parameters $\zeta$ and $\delta$ are positive.

Theorem 3.2. Using the control law (3.37), the states of the closed-loop system (3.20) converge to the chosen value in finite time.

Proof. Let the positive-definite Lyapunov functional $V_{0}$ be

$$
V_{0}=\frac{1}{2} s_{t}^{T} s_{t} .
$$

Using Eqs. (3.36) and (3.37), the time derivative of the Lyapunov function is given by

$$
\begin{aligned}
\dot{V}_{0} & =s_{t}^{T} \dot{s}_{t} \\
& =s_{t}^{T}\left(f(x)+g(x) u_{c}-x_{d}+\dot{s}_{d}\right) \\
& =s_{t}^{T}\left(f(x)-\left(f(x)-\dot{x}_{d}+\delta s_{t}+\zeta s_{t}^{p / q}+\hat{d}\right)-\dot{x}_{d}+\dot{s}_{d}\right) \\
& =s_{t}^{T}\left(-\delta s_{t}-\zeta s_{t}^{p / q}+d-\hat{d}+\dot{s}_{d}\right) .
\end{aligned}
$$

According to Eq. (3.28), $d-\hat{d}=-\tilde{d}=-\dot{s}_{d}$. Hence Eq. (3.39) can be rewritten as

$$
s_{t}^{T} \dot{s}_{t}=-s_{t}^{T}\left(\delta s_{t}+\zeta s_{t}^{p / q}\right) .
$$

From Lemma 3.1, the proposed control scheme fulfills the Lyapunov condition, and it can be confirmed that the states of the closed-loop system converge to the desired value in finite time.

Remark 3.3. In this study, the stability of the closed-loop system has been proven. Therefore, based on presented stability in Theorem 3.1 and Theorem 3.2, when variations of the system parameters and its initial conditions result in bounded uncertainties and disturbances the proposed control is stable, and states of the systems converge to their desired value in finite time.

\subsection{Fuzzy inference logic}

In the proposed control technique, instead of discontinuous function $\operatorname{sign}($.$) , the fuzzy engine is applied to$ reduce the chattering phenomena and achieve the continuously smooth control input. In order to suppress 
the chattering, some research studies offere to use a saturation function $\operatorname{sat}(s / \phi)$ instead of $\operatorname{sign}().[4]$. The saturation function is given by

$$
\operatorname{sat}(s / \phi)=\left\{\begin{array}{l}
\operatorname{sign}(s / \phi), \text { if }|s / \phi|>1 \\
s / \phi, \text { if }|s / \phi| \leq 1
\end{array}\right.
$$

where parameter $\phi$ is the boundary layer thickness which is defined around the sliding surface. However, this method may add a steady-state error into the sliding manifold [31]. Since the boundary layer method is employed to avoid the chattering phenomena, there exists a tradeoff between the control smoothness and tracking error. To overcome this issue, in the current research, the fuzzy logic system has been proposed to reduce or eliminate the chattering without sacrificing the tracking accuracy. In the literature, no study proposes a fast fuzzy disturbance observer-based TSMC with guaranteed finite-time convergence on the basis of this approach for chattering annihilation.

The fuzzy logic maps the input variables $s_{d}$ and $\dot{s}_{d}$ to the output variable $F_{s d}$, where $F_{s d}$ is used instead of the sign $\left(s_{d}\right)$. The fuzzy rules are chosen in such a way that fulfills the stability conditions. In fact, based on the stability conditions of the closed-loop system, the positivity or negativity of $F_{s d}$ is taken from sign $\left(s_{d}\right)$. In the current study, the Mamdani fuzzy system is used. Fig. 5 shows the Gaussian membership functions which are utilized for linguistic variables. Five fuzzy partitions were selected for variables $F_{s d}, s_{d}$, and $\dot{s}_{d}$, where the symbols for these fuzzy partitions are NB(Negative Big), N(Negative), $\mathrm{Z}$ (Zero), and $\mathrm{P}$ (Positive), $\mathrm{PB}$ (Positive Big). The fuzzy sets are also normalized in the interval $(-1,1)$. The fuzzy rules, implemented in this study, are given in Table 1.

For the fuzzy interference engine, the value of $F_{s d}$ will be obtained adaptively. The procedure for the fuzzy disturbance-observer-based TSMC is illustrated in Fig.6. The fast disturbance is the observer, and the fuzzy system have been combined with TSMC to enhance the effectiveness of the controller in the presence of an external disturbance and unexpected uncertainty. In the next section, the proposed control scheme will be applied to the 5D self-exciting homopolar disc dynamo system.

\section{Simulation results}

In this section, we show the simulation results of synchronization using the designed fuzzy disturbanceobserver-based TSMC for the quintic self-exciting homopolar disc dynamo. The equations for the slave system with control input and external disturbance are given by

$$
\left\{\begin{array}{l}
\dot{y}_{1}=r\left(y_{2}-y_{1}\right)+y_{4}+d_{1}+u_{1} \\
\dot{y}_{2}=-(1+m) y_{2}+y_{1} y_{3}-y_{5}+d_{2}+u_{2} \\
\dot{y}_{3}=g\left[1+m y_{1}^{2}-(1+m) y_{1} y_{2}\right]+d_{3}+u_{3} \\
\dot{y}_{4}=2(1+m) y_{4}+y_{1} y_{3}-k_{1} y_{1}+d_{4}+u_{4} \\
\dot{y}_{5}=-m y_{5}+k_{2} y_{2}+d_{5}+u_{5}
\end{array}\right.
$$

Based on the design procedure, presented in the previous section, the control signal and estimated disturbance for this system are given by

$$
\begin{aligned}
& u_{c}=-g^{-1}(Y)\left(f(Y)-\dot{Y}_{d}+\delta s_{t}+\zeta s_{t}^{p / q}+\hat{d}\right), \\
& \hat{d}=-k s_{d}-\beta F_{s d}-\varepsilon s_{d}^{p / q}-|f(Y)| F_{s d}-f(Y) .
\end{aligned}
$$

Here $F_{s d}$ is the output of the fuzzy engine. Vectors

$$
Y=\left[y_{1}, y_{2}, y_{3}, y_{4}, y_{5}\right], u_{c}\left[u_{1}, u_{2}, u_{3}, u_{4}, u_{5}\right], \hat{d}=\left[\hat{d}_{1}, \hat{d}_{2}, \hat{d}_{3}, \hat{d}_{4}, \hat{d}_{5}\right]
$$

represent the state of the system, the control signal, and the estimated disturbance, respectively. The master system is

$$
\left\{\begin{array}{l}
\dot{x}_{1}=r\left(x_{2}-x_{1}\right)+x_{4} \\
\dot{x}_{2}=-(1+m) x_{2}+x_{1} x_{3}-x_{5} \\
\dot{x}_{3}=g\left(1+m x_{1}^{2}-(1+m) x_{1} x_{2}\right), \\
\dot{x}_{4}=2(1+m) x_{4}+x_{1} x_{3}-k_{1} x_{1} \\
\dot{x}_{5}=-m x_{5}+k_{2} x_{2}
\end{array}\right.
$$


The parameters for the slave and master systems are $r=0.8, m=0.04, g=140.6, k_{1}=34$, and $k_{2}=12$, while the control parameters are

$$
\begin{array}{ll}
q=[13,13,13,13,13], \quad k=[10,10,10,10,10], \quad \beta=[100,100,100,100,100], \\
\varepsilon=[10,10,10,10,10], \quad p_{0}=[3,3,3,3,3], \quad q_{0}=[9,9,9,9,9]
\end{array}
$$

To show the performance of the controller, we suppose that we are given incorrect information about the slave system parameters with these parameters set at $50 \%$ of their actual values:

$$
\hat{r}=0.5 \times r, \hat{m}=0.5 \times r, \hat{g}=0.5 \times g, \hat{k_{1}}=0.5 \times k_{1}, \hat{k_{2}}=0.5 \times k_{2} .
$$

Thus $\hat{r}, \hat{m}, \hat{g}, \hat{k_{1}}$, and $\hat{k_{2}}$, are the incorrect parameter values, and are used for the control scheme instead of $\hat{r}, \hat{m}, \hat{g}, \hat{k_{1}}$, and $\hat{k_{2}}$. Moreover, the system has an unknown external disturbance as follows:

$$
d_{i}(t)=0.1 \sin (t)+0.2 \cos (2 t) .
$$

The initial condition of slave and master systems are chosen as $(1.25,-2,4.4,5.2,7.7)$ and $(0,-0.5,0.1$, $-1,-1)$, respectively. The input control are applied at $t=3$. The synchronization results are shown in Figs. 7-9. Tracking of the master system states via the slave system under the proposed controller is illustrated in Fig. 7. Fig. 8 shows the time series of synchronization errors. After less than one time unit, synchronization is achieved with the slave system mimicing the master system. Moreover, as these figures show, since the proposed controller is equipped with a fuzzy engine, the states of the system are chatter-free with smooth responses. The time series of the control signals is shown in Fig. 9.

Numerical simulations therefore confirm that the fuzzy disturbance-observer-based designed TSMC successfully drives the system states to their desired value, even when the information of the slave system is inaccurate and there exist external disturbances. To conclude, the results of synchronization show that the control technique we have developed in this paper can serve as a robust control technique for uncertain nonlinear systems, thereby ensuring that such systems operate chatter-free in the desired mode.

\section{Conclusion}

In the first part of this study, a Hopf bifurcation from a stable equilibrium state for a fifth order selfexciting homopolar disc dynamo have been studied. We extended our analytical results of [41], to find two unstable small amplitude limit cycles for two isolated Hopf equilibria. The new results will be useful for explain multistability for the chosen parameter and initial values. They show that those unstable periodic solutions will present statistical properties of the hidden chaotic solutions. Next, a new fuzzy disturbance-observer based terminal sliding mode control was designed for the purposes of synchronization. The output of the fast disturbance-observer was combined with terminal sliding mode control for the chaotic system by considering external disturbances and unexpected uncertainties. To avoid the chattering problem, the designed control scheme was improved by implementing a fuzzy interference engine. Numerical simulations clearly show the performance of the designed controller for the five-dimensional hyperchaotic system. It will make sense to consider that the proposed control technique could be extended with fractional-order calculus.

\section{Acknowledgements}

This work was supported by the National Natural Science Foundation of China (Nos. 11772306,11832002), Zhejiang Provincial Natural Science Foundation of China under Grant (No.LY20A020001), and the Fundamental Research Funds for the Central Universities, China University of Geosciences (CUGGC05).

\section{References}

[1] Aghababa M.P., \& Heydari A. [2012] "Chaos synchronization between two different chaotic systems with uncertainties, external disturbances, unknown parameters and input nonlinearities," Applied Mathematical Modelling 36, 1639-1652. 
[2] Bao B. C., Li Q. D., Wang N., \& Xu Q. [2016] "Multistability in Chua's circuit with two stable node-foci," Chaos: An Interdisciplinary Journal of Nonlinear Science 26, 043111.

[3] Bao B. C., Luo J., Bao H., Chen C., Wu H., \& Xu Q. [2019] "A Simple Nonautonomous Hidden Chaotic System with a Switchable Stable Node-Focus," International Journal of Bifurcation and Chaos 29, 1950168.

[4] Chen M., \& Chen W.H. [2010] "Sliding mode control for a class of uncertain nonlinear system based on disturbance observer," International Journal of Adaptive Control and Signal Processing 24,51-64.

[5] Chen M., Wu Q. X., \& Cui R. X. [2013] "Terminal sliding mode tracking control for a class of SISO uncertain nonlinear systems," ISA transactions 52, 198-206.

[6] Dudkowski D., Jafari S., Kapitaniak T., Kuznetsov N. V., Leonov G. A., \& Prasad A. [2016] "Hidden attractors in dynamical systems," Physics Reports 637, 1-50.

[7] Guerrero J., Torres J., Creuze V., \& Chemori A. [2020] "Adaptive disturbance observer for trajectory tracking control of underwater vehicles," Ocean Engineering 200, 107080.

[8] Huang G. [2010] "A fast terminal sliding mode control with novel fuzzy disturbance observer," 2010 International Conference on Web Information Systems and Mining, Sanya, 95-99.

[9] Jahanshahi H. [2018] "Smooth control of HIV/AIDS infection using a robust adaptive scheme with decoupled sliding mode supervision," The European Physical Journal Special Topics 227, 707-718.

[10] Jahanshahi H., Rajagopal K., Akgul A., Sari N. N., Namazi H., \& Jafari S. [2018] "Complete analysis and engineering applications of a megastable nonlinear oscillator," International Journal of Non-Linear Mechanics 107, 126-136.

[11] Jahanshahi H., Sari N.N., Pham V-T., Alsaadi F.E., \& Hayat T. [2018] "Optimal adaptive higher order controllers subject to sliding modes for a carrier system," International Journal of Advanced Robotic Systems 15, 1729881418782097.

[12] Jahanshahi H., Shahriari-Kahkeshi M., Alcaraz R., Wang X., Singh V. P., \& Pham V. T. [2019] "Entropy analysis and neural network-based adaptive control of a non-equilibrium four-dimensional chaotic system with hidden attractors," Entropy 21, 156.

[13] Jahanshahi H., Yousefpour A., Munoz-Pacheco J. M., Moroz I., Wei Z., \& Castillo O. [2020] "A new multi-stable fractional-order four-dimensional system with self-excited and hidden chaotic attractors: Dynamic analysis and adaptive synchronization using a novel fuzzy adaptive sliding mode control method," Applied Soft Computing 87, 105943.

[14] Leonov G. A., Kuznetsov N. V., \& Vagaitsev V. I. [2011] "Localization of hidden Chua?s attractors," Physics Letters A 375, 2230-2233.

[15] Leonov G. A., Kuznetsov N. V., \& Vagaitsev V. I. [2012] "Hidden attractor in smooth Chua systems" Physica D: Nonlinear Phenomena 241, 1482-1486.

[16] Li C., \& Sprott J. C. [2018] "An infinite 3-D quasiperiodic lattice of chaotic attractors," Physics Letters A 382, 581-587.

[17] Li S., Yang J., Chen W.H., \& Chen X. [2016] "Disturbance observer-based control: methods and applications: CRC press,".

[18] Liu H., Bao W., Li H., \& Liao Y. [2019] "Multivariable disturbance observer-based fuzzy fast terminal sliding mode attitude control for a hypersonic vehicle," Journal of Aerospace Engineering 32, 04018152.

[19] Ma J., Zhang G., Hayat T., \& Ren G. [2019] "Model electrical activity of neuron under electric field," Nonlinear Dynamics 95, 1585-1598.

[20] Mahmoodabadi M. J., \& Jahanshahi H. [2016] "Multi-objective optimized fuzzy-PID controllers for fourth order nonlinear systems," Engineering Science and Technology, an International Journal 19, 1084-1098.

[21] Moffatt H. K. [1979] "A self-consistent treatment of simple dynamo systems," Geophysical \& Astrophysical Fluid Dynamics 14, 147-166.

[22] Mobayen S. [2018] "Design of novel adaptive sliding mode controller for perturbed Chameleon hidden chaotic flow," Nonlinear Dynamics 1-15.

[23] Nazarimehr F., Jafari S., Hashemi Golpayegani S. M. R., Perc M., \& Sprott J. C. [2018] "Predicting tipping points of dynamical systems during a period-doubling route to chaos," Chaos: An Interdisciplinary Journal of Nonlinear Science 28, 073102. 
[24] Nguyen S.D., Choi S.B., \& Nguyen Q.H. [2018] "A new fuzzy-disturbance observer-enhanced sliding controller for vibration control of a train-car suspension with magneto-rheological dampers," Mechanical Systems and Signal Processing 105, 447-466.

[25] Ni J., Liu L., Liu C., Hu X., \& Li S. [2016] "Fast fixed-time nonsingular terminal sliding mode control and its application to chaos suppression in power system," IEEE Transactions on Circuits and Systems II: Express Briefs 64, 151-155.

[26] Rajagopal K., Akgul A., Pham V. T., Alsaadi F. E., Nazarimehr F., Alsaadi F. E., et al. [2019] "Multistability and coexisting attractors in a new circulant chaotic system," International Journal of Bifurcation and Chaos 29, 1950174.

[27] Rajagopal K., Jahanshahi H., Jafari S., Weldegiorgis R., Karthikeyan A., \& Duraisamy P. [2020] "Coexisting attractors in a fractional order hydro turbine governing system and fuzzy PID based chaos control," Asian Journal of Control DOI: 10.1002/asjc.2261.

[28] Rajagopal K., Jahanshahi H., Varan M., Bay?r I., Pham V. T., Jafari S., et al. [2018] "A hyperchaotic memristor oscillator with fuzzy based chaos control and LQR based chaos synchronization," $A E U$ International Journal of Electronics and Communications 94, 55-68.

[29] Sari N. N., Jahanshahi H., \& Fakoor M. [2019] "Adaptive fuzzy PID control strategy for spacecraft attitude control," International Journal of Fuzzy Systems 21, 769-781.

[30] Shahzad M., Pham V.T., Ahmad M.A., Jafari S., \& Hadaeghi F. [2015] "Synchronization and circuit design of a chaotic system with coexisting hidden attractors," The European Physical Journal Special Topics 224, 1637-1652.

[31] Slotine J.J.E. \& Li W. [1991] Applied Nonlinear Control. Englewood Cliffs, Prentice Hall, New Jersey.

[32] Soradi-Zeid S., Jahanshahi H., Yousefpour A, \& Bekiros S. [2020] "King algorithm: A novel optimization approach based on variable-order fractional calculus with application in chaotic financial systems," Chaos, Solitons \& Fractals 132, 109569.

[33] Sprott J. C. [1994] "Some simple chaotic flows," Physical Review E 50 R647.

[34] Sun J., Wang Y., Wang Y., \& Shen Y. [2016] "Finite-time synchronization between two complexvariable chaotic systems with unknown parameters via nonsingular terminal sliding mode control," Nonlinear Dynamics 85, 1105-1117.

[35] Tao C., \& Liu X. [2007] "Feedback and adaptive control and synchronization of a set of chaotic and hyperchaotic systems," Chaos, Solitons $\&$ Fractals 32, 1572-1581.

[36] Vahidi-Moghaddam A., Rajaei A., \& Ayati M. [2019] "Disturbance-observer-based fuzzy terminal sliding mode control for MIMO uncertain nonlinear systems," Applied Mathematical Modelling 70, 109-127.

[37] Wang S., Yousefpour A., Yusuf A., Jahanshahi H., Alcaraz R., He S., et al. [2020] "Synchronization of a Non-Equilibrium Four-Dimensional Chaotic System Using a Disturbance-Observer-Based Adaptive Terminal Sliding Mode Control Method," Entropy 22, 271.

[38] Wei X.J., Wu Z.J., \& Karimi H.R. [2016] "Disturbance observer-based disturbance attenuation control for a class of stochastic systems," Automatica 63, 21-25.

[39] Wei Z., Li Y., Sang B., Liu Y., \& Zhang W. [2019] "Complex dynamical behaviors in a 3D simple chaotic flow with 3D stable or 3D unstable manifolds of a single equilibrium," International Journal of Bifurcation and Chaos 29, 1950095.

[40] Wei Z., Moroz I., Sprott J. C., Wang Z., \& Zhang W. [2017] "Detecting hidden chaotic regions and complex dynamics in the self-exciting homopolar disc dynamo," International Journal of Bifurcation and Chaos 27, 1730008.

[41] Wei Z., Moroz I., Sprott J. C., Akgul A., \& Zhang W. [2017] "Hidden hyperchaos and electronic circuit application in a 5D self-exciting homopolar disc dynamo," Chaos: An Interdisciplinary Journal of Nonlinear Science 27, 033101.

[42] Wei Z., \& Zhang W. [2014] "Hidden hyperchaotic attractors in a modified LorenzCStenflo system with only one stable equilibrium," International Journal of Bifurcation and Chaos 24, 1450127.

[43] Wu F., Hayat T., An X., \& Ma J. [2018] "Can Hamilton energy feedback suppress the chameleon chaotic flow?" Nonlinear Dynamics 94, 669-677.

[44] Yassen M. T. [2007] "Controlling, synchronization and tracking chaotic Liu system using active backstepping design," Physics Letters A. 360, 582-587. 
[45] Yousefpour A., Jahanshahi H., Munoz-Pacheco J.M., Bekiros S., \& Wei Z. [2020] "A fractional-order hyper-chaotic economic system with transient chaos," Chaos, Solitons \& Fractals 130, 109400.

[46] Yousefpour A., \& Jahanshahi H. [2019] "Fast disturbance-observer-based robust integral terminal sliding mode control of a hyperchaotic memristor oscillator," The European Physical Journal Special Topics 228, 2247-2268.

[47] Yang Q., Wei Z., \& Chen G. [2010] "An unusual 3D autonomous quadratic chaotic system with two stable node-foci," International Journal of Bifurcation and Chaos 20, 1061-1083.

[48] Yu X., \& Zhi H. M. [2002] "Fast terminal sliding-mode control design for nonlinear dynamical systems," IEEE Transactions on Circuits and Systems I: Fundamental Theory and Applications 49, 261-264.

[49] Zhang J., Zhao W., Shen G., \& Xia Y. [2020] "Disturbance observer-based adaptive finite-time attitude tracking control for rigid spacecraft," IEEE Transactions on Systems, Man, and Cybernetics: Systems. DOI: 10.1109/TSMC.2019.2947320.

[50] Zhou T., \& Chen G. [2006] "Classification of chaos in 3-D autonomous quadratic systems-I: basic framework and methods," International Journal of Bifurcation and Chaos 16, 2459-2579. 


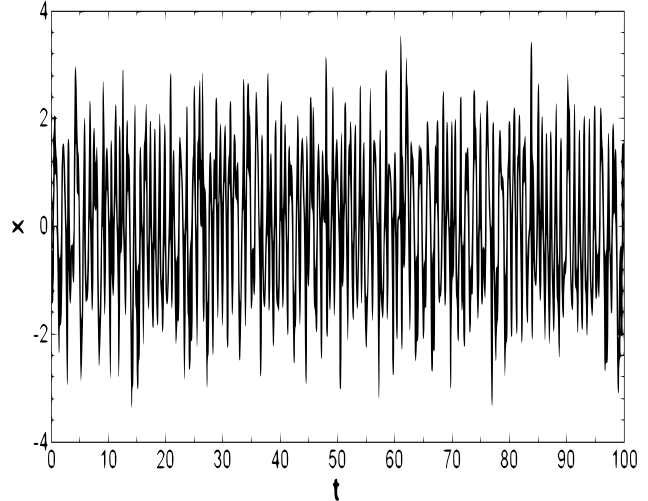

(a)

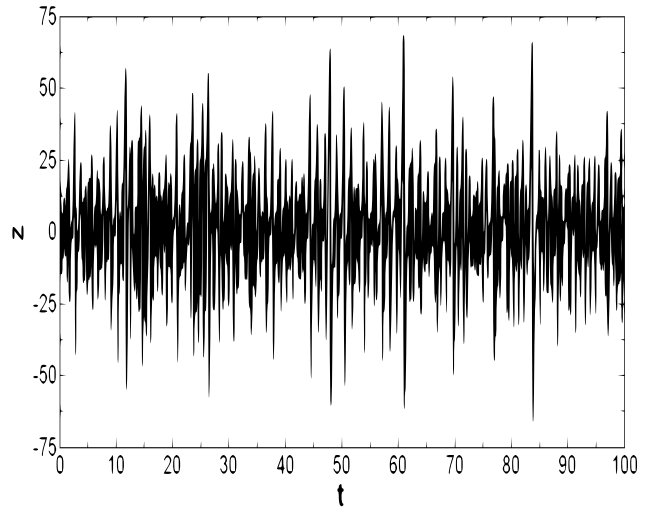

(c)

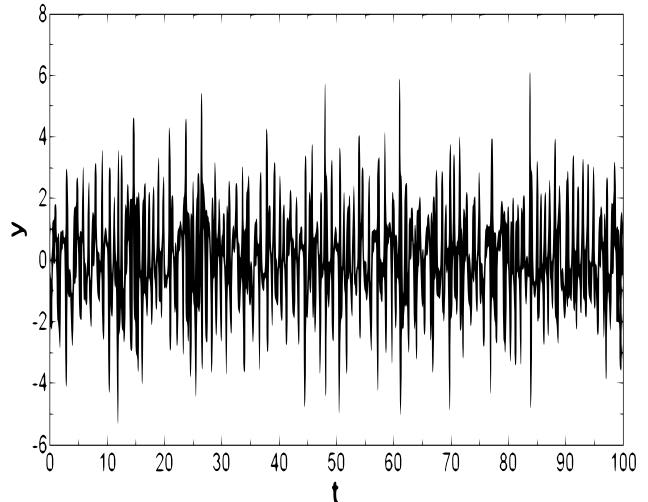

(b)

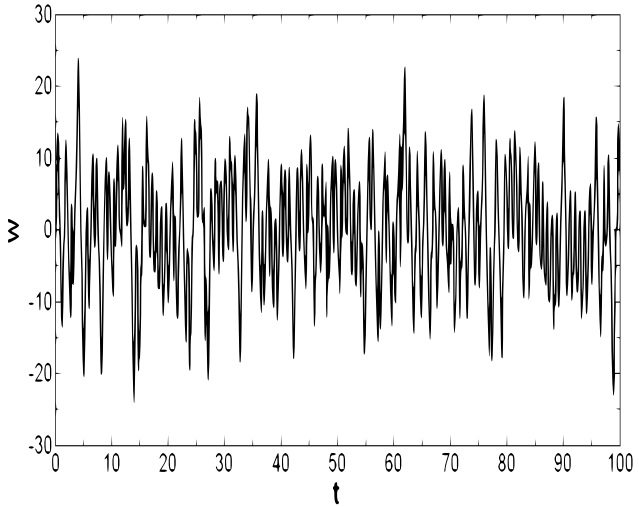

(d)

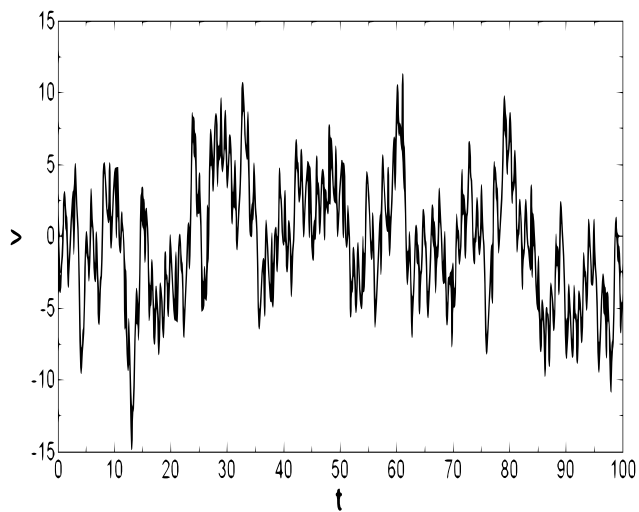

(e)

Figure 1: When $r=8, m=0.04, g=140.6, k_{1}=34$ and $k_{2}=12$, the initial state is taken as $(0.05,-0.5$, $0.1,-1,2)$. We show the following time series for system (1): (a) $x(t)$; (b) $y(t) ;(\mathrm{c}) z(t)$; (d) $w(t)$; (e) $v(t)$. 


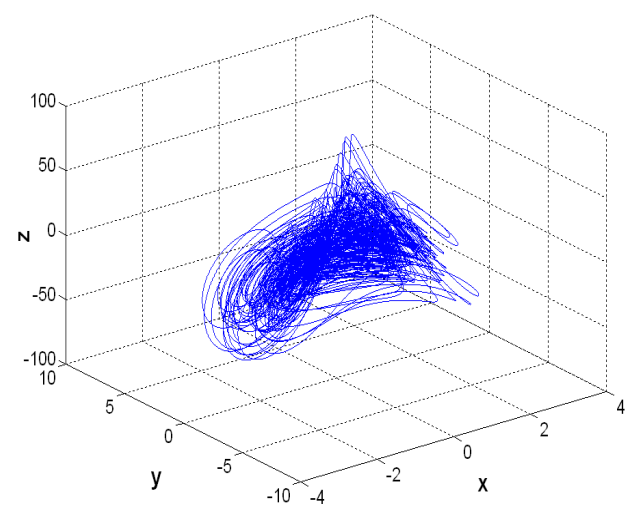

(a)

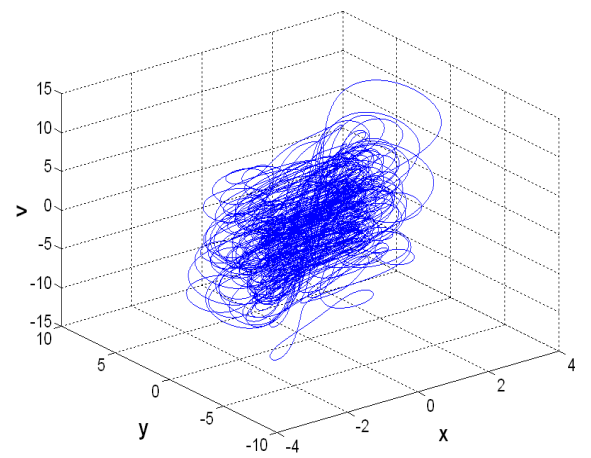

(c)

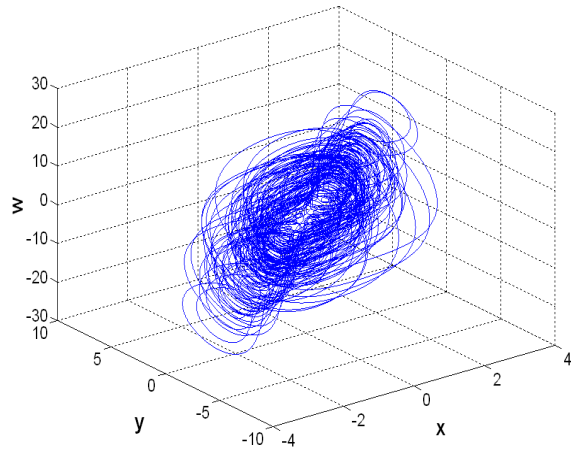

(b)

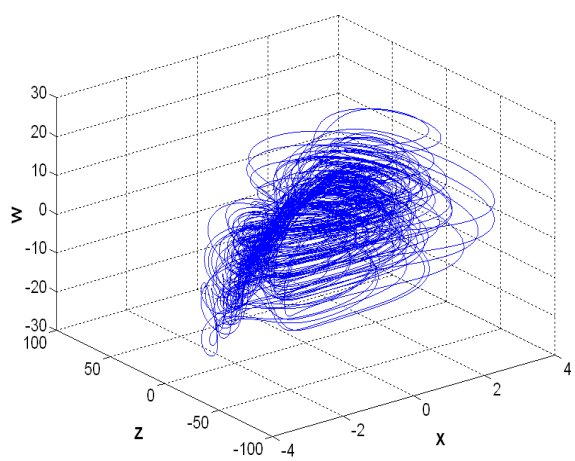

(d)

Figure 2: When $r=8, m=0.04, g=140.6, k_{1}=34$ and $k_{2}=12$, the initial state is taken as $(0.05,-0.5$, $0.1,-1,2)$. We show the following different phases for system (1): (a) $x(t)-y(t)-z(t)$; (b) $x(t)-y(t)-w(t)$; (c) $x(t)-y(t)-v(t)$; (d) $x(t)-z(t)-w(t)$.
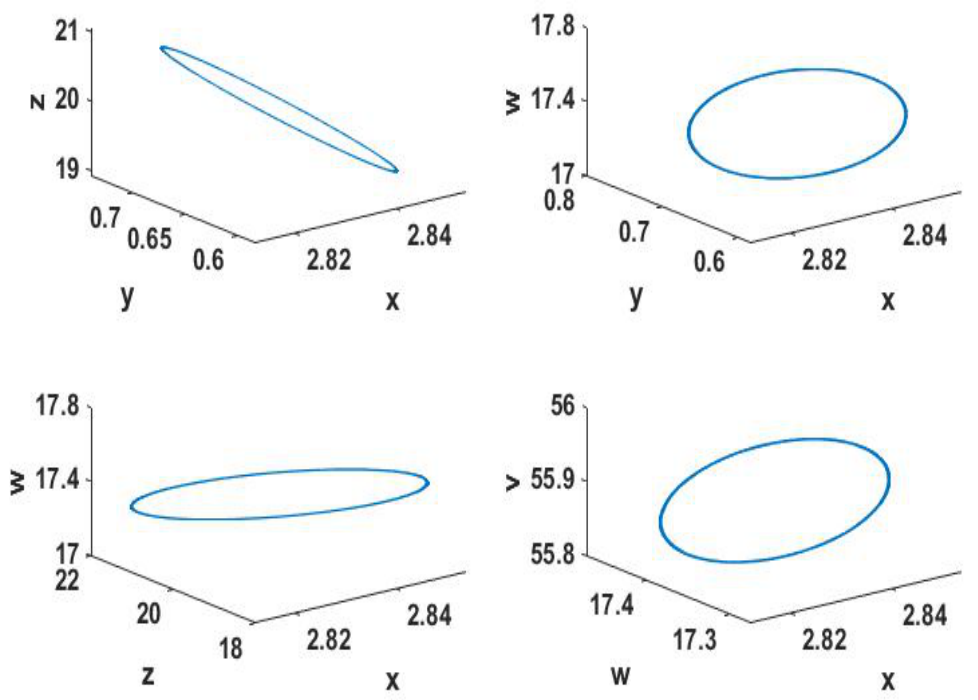

Figure 3: When initial condition $(2.8,1,20,16.7,55)$ and parameters $r=8, m=0.142, g=140.6, k_{1}=34$ and $k_{2}=12$, system (1) shows an unstable periodic orbit. 

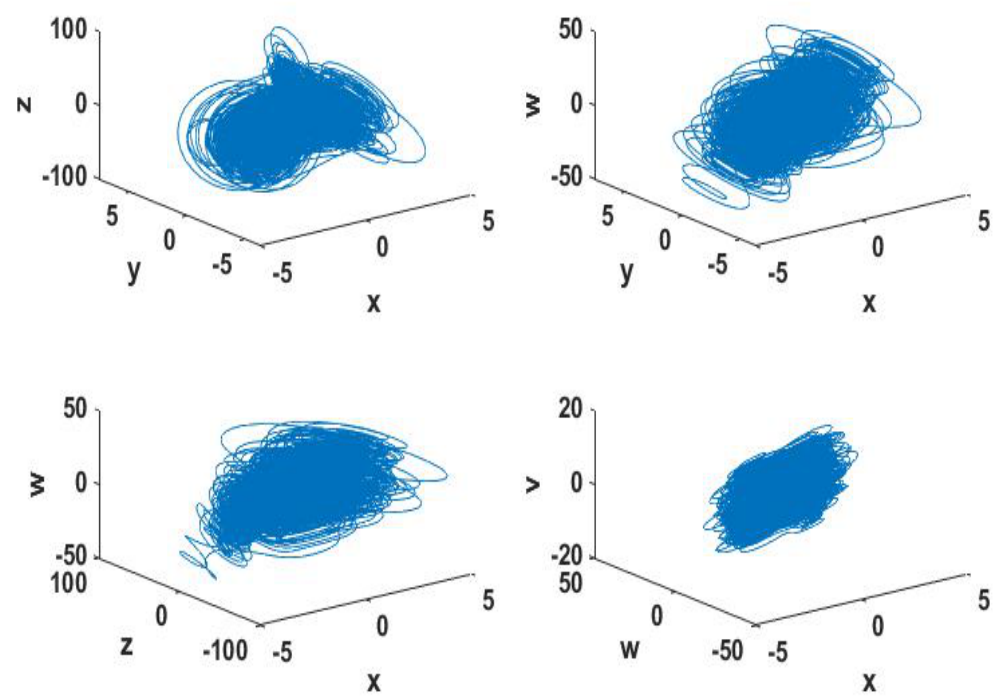

Figure 4: When initial condition $(1,0,0,0,4.9)$ and parameters $r=8, m=0.142, g=140.6, k_{1}=34$ and $k_{2}=12$, system (1) shows chaotic attractor with only two stable equilibrium points.

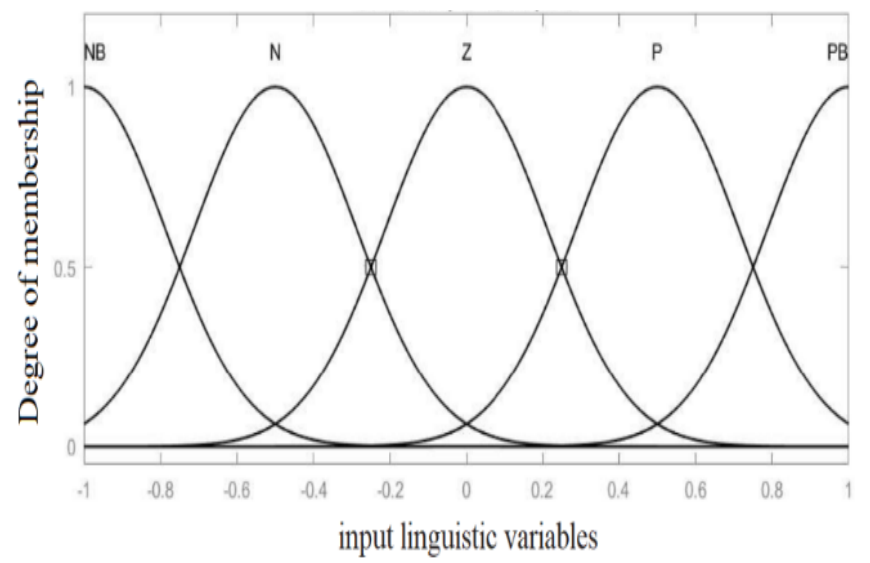

(a)

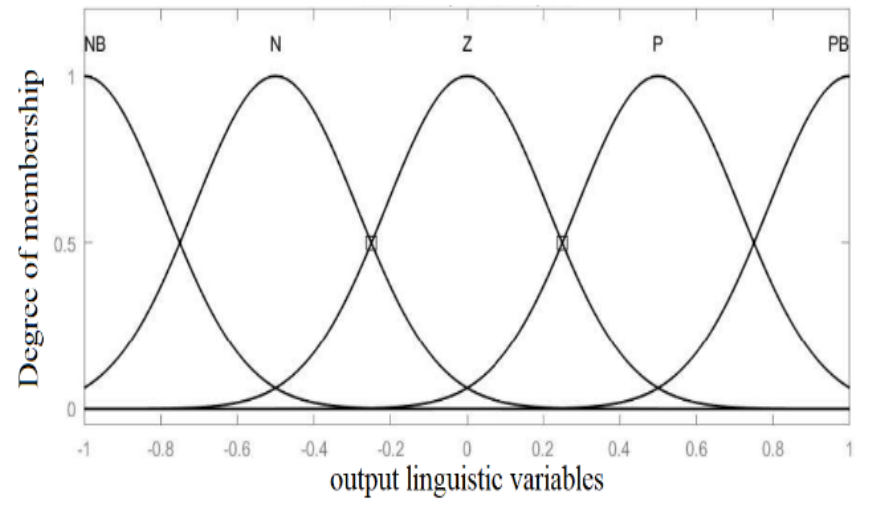

(b)

Figure 5: Membership functions: (a) input linguistic variables $s_{d}$ and $\dot{s}_{d}$; (b) output linguistic variables $F_{s d}$. 
Table 1: Fuzzy rule base

\begin{tabular}{|l|l|l|l|l|l|l|}
\hline & \multicolumn{7}{|c|}{$\dot{\boldsymbol{s}}_{\boldsymbol{d}}$} \\
\hline \multirow{2}{*}{$\boldsymbol{F}_{\boldsymbol{s} \boldsymbol{d}}$} & $\mathrm{NB}$ & $\mathrm{N}$ & $\mathrm{Z}$ & $\mathrm{P}$ & $\mathrm{PB}$ \\
\hline \multirow{3}{*}{$\boldsymbol{s}_{\boldsymbol{d}}$} & $\mathrm{NB}$ & $\mathrm{NB}$ & $\mathrm{NB}$ & $\mathrm{N}$ & $\mathrm{N}$ & $\mathrm{N}$ \\
\hline & $\mathrm{N}$ & $\mathrm{NB}$ & $\mathrm{N}$ & $\mathrm{N}$ & $\mathrm{N}$ & $\mathrm{N}$ \\
\hline & $\mathrm{Z}$ & $\mathrm{Z}$ & $\mathrm{Z}$ & $\mathrm{Z}$ & $\mathrm{Z}$ & $\mathrm{Z}$ \\
\hline & $\mathrm{P}$ & $\mathrm{P}$ & $\mathrm{P}$ & $\mathrm{P}$ & $\mathrm{P}$ & $\mathrm{PB}$ \\
\hline & $\mathrm{PB}$ & $\mathrm{P}$ & $\mathrm{P}$ & $\mathrm{P}$ & $\mathrm{PB}$ & $\mathrm{PB}$ \\
\hline
\end{tabular}

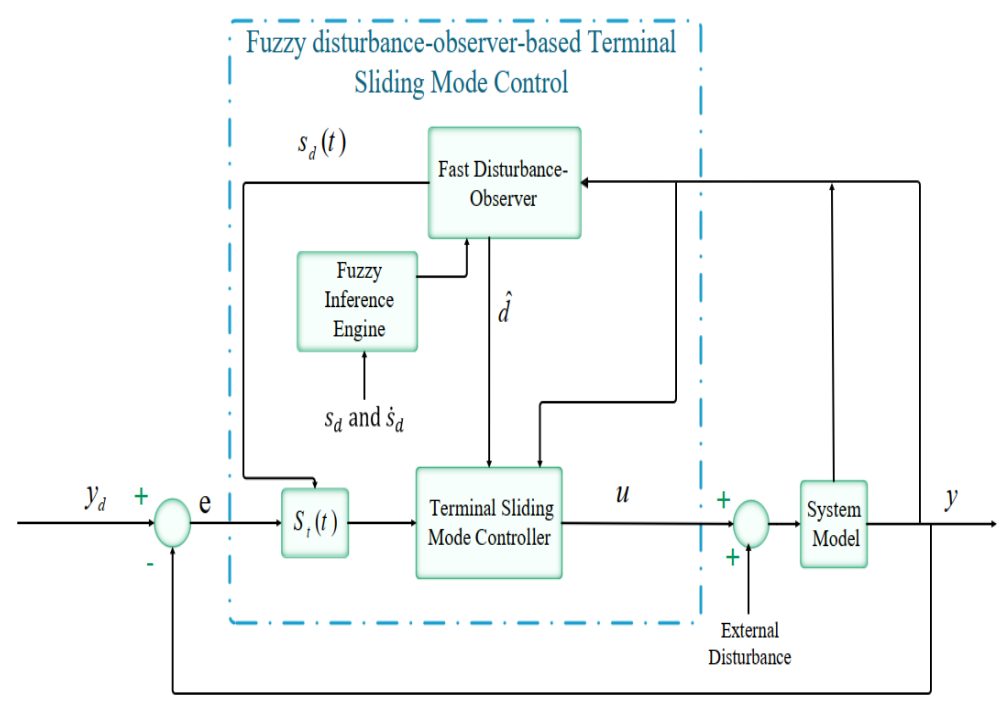

Figure 6: Block diagram of fuzzy disturbance-observer-based TSMC for a 5D self-exciting homopolar disc dynamo system. 

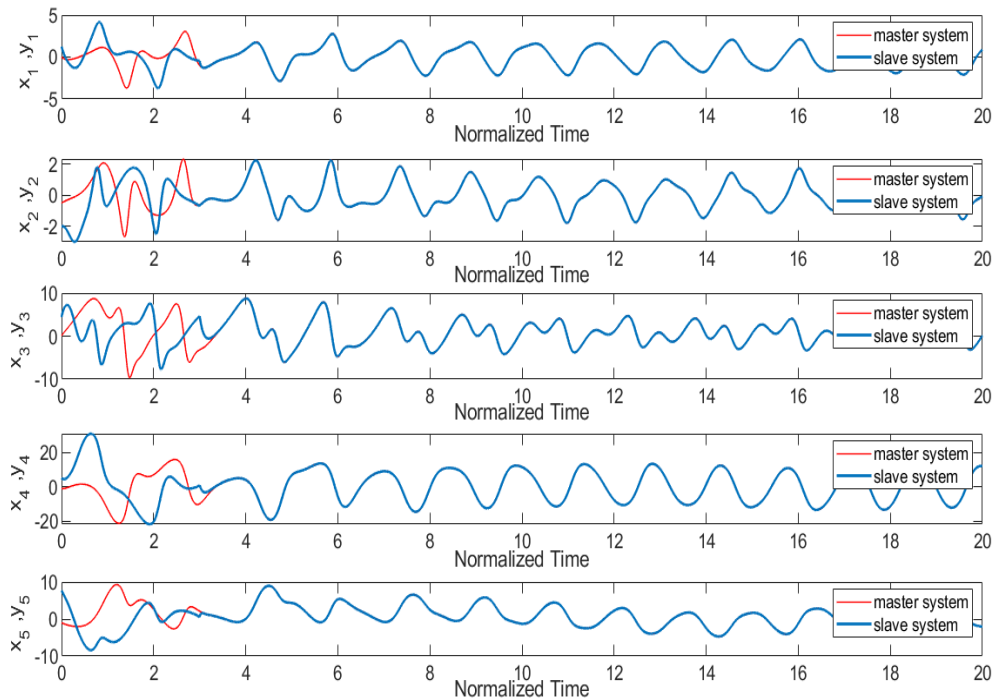

Figure 7: The time series of the synchronization for the five-dimensional system based on the fuzzy disturbance-observer-based TSMC.
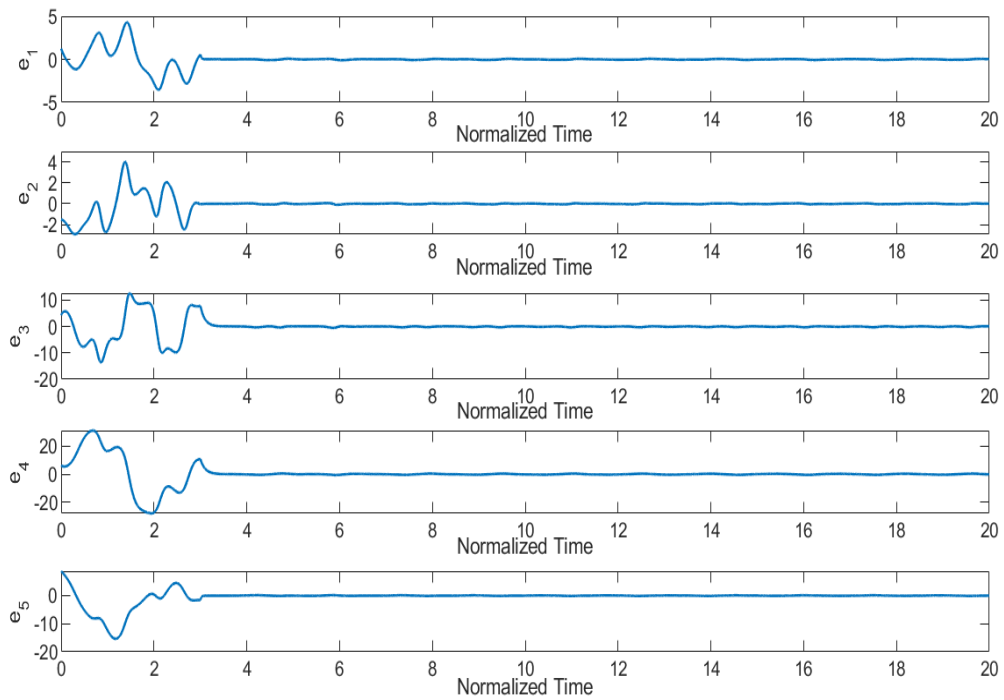

Figure 8: Synchronization error of the five-dimensional hyperchaotic system based on the fuzzy disturbance-observer-based TSMC. 

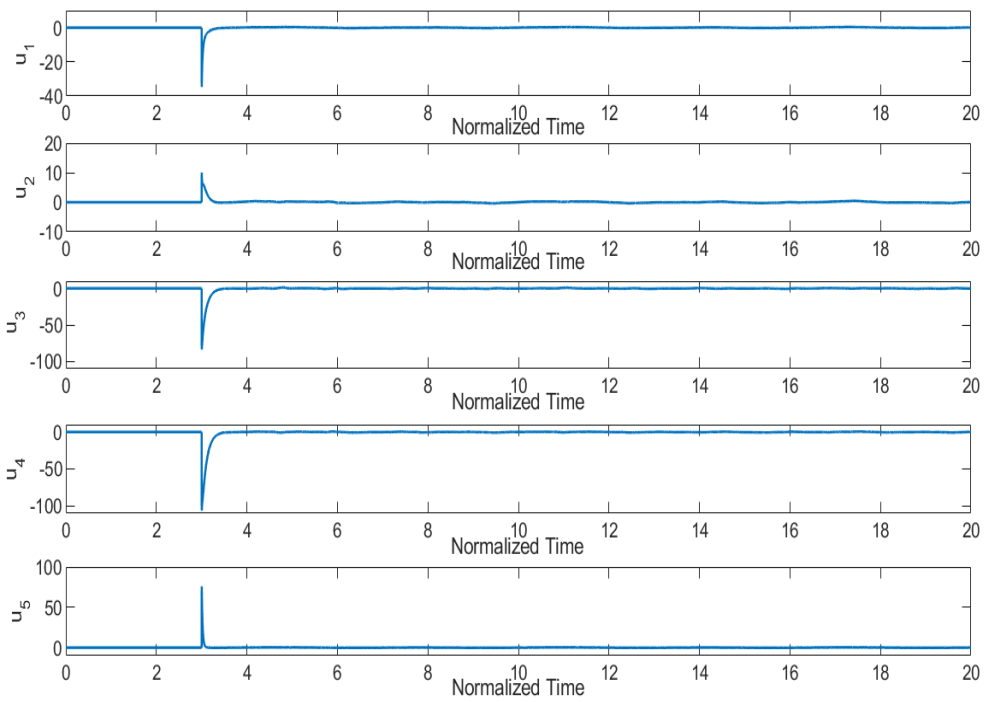

Figure 9: The control input based on the fuzzy disturbance-observer-based TSMC. 